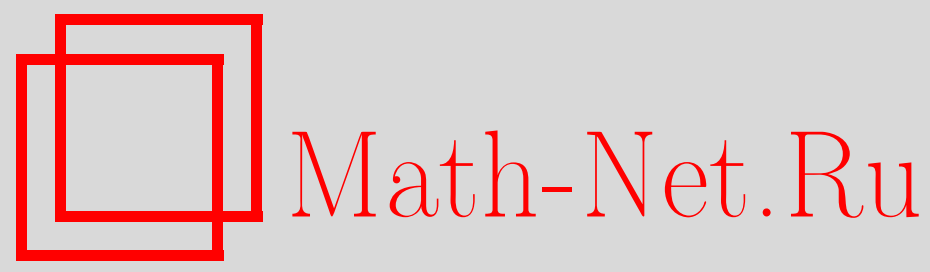

Ю. С. Волков, О неотрицательном решении системы уравнений с симметрической циркулянтной матрицей, Матем. заметки, 2001, том 70, выпуск 2, 170-180

DOI: https://doi.org/10.4213/mzm731

Использование Общероссийского математического портала Math-Net.Ru подразумевает, что вы прочитали и согласны с пользовательским соглашением http://www . mathnet.ru/rus/agreement

Параметры загрузки:

IP : 54.210 .77 .194

26 апреля 2023 г., $16: 27: 53$

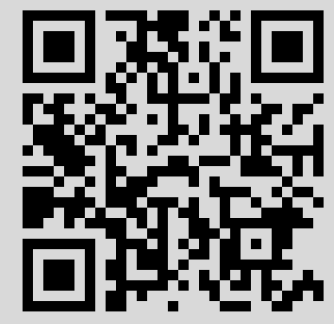




\title{
О НЕОТРИЦАТЕЛЬНОМ РЕШЕНИИ СИСТЕМЫ УРАВНЕНИЙ С СИММЕТРИЧЕСКОЙ ЦИРКУЛЯНТНОЙ МАТРИЦЕЙ
}

\author{
Ю. С. Волков
}

\begin{abstract}
Для системы уравнений с симметрической циркулянтной матрицей найдены условия на вектор правой части, обеспечиваюшие положительность решения системы. В качестве приложения полученньх результатов исследована задача положительной сплайн-интерполяции положительных функций на равномерных сетках.

Библиографоия: 12 названий.
\end{abstract}

Стандартные процессы интерполящии сплайнами обычно требуют решения линейных систем вида

$$
A x=d
$$

с ленточной матрицей коэффищиентов $A$. Необходимость наследования интерполянтом таких характеристик исходных данньх, как положительность, монотонность или выпуклость, приводит к необходимости изучения вопроса о положительном (неотрищательном) решении системы уравнений (1) при положительной правой части $d$.

При исследовании условий монотонности и выпуклости кубических и параболических сплайнов Мирошниченко [1] применил специальное преобразование трехдиагональной системы уравнений с неотрицательными коэффициентами, приводяшее к системе с матрицей монотонного вида (матрица $M$ монотонна, если из условия $M x \geqslant 0$ следует $x \geqslant 0$ ). Эта идея оказалась очень полезной и плодотворной, она нашла широкое применение при получении достаточных условий монотонности и/или выпуклости для различных обобщений кубических сплайнов [2]-[4]. В дальнейшем Завьялов [5] в развитие идеи Мирошниченко получил условие неотрицательности решения системы (1) для якобиевых матрищ. Однако для матриц с шириной ленты больше 3 такой подход уже не работает.

В данной заметке мы будем считать, что ленточная матрица $A$ системы $(1)$ является циркулянтной

$$
A=\operatorname{circ}\left(a_{n}, a_{n-1}, \ldots, a_{1}, 1,0, \ldots, 0,1, a_{1}, \ldots, a_{n-1}\right),
$$

где первая определяющая строка [6] матрицы $A$ имеет вид $\left(a_{n}, a_{n-1}, \ldots, a_{1}, 1,0, \ldots, 0\right.$, $\left.1, a_{1}, \ldots, a_{n-1}\right)$. В задачах сплайн-интерполяции система $(1)$ с такой матрищей возникает при периодической интерполяции на равномерной сетке [7]. 
Цель работы состоит в нахождении невырожденной квадратной матрицы $G$ такой, что матрица $M=G A$ будет монотонной. Тогда для системы (1) условием, гарантируюшим неотрицательность решения, будет

$$
G d \geqslant 0
$$

Кроме того, нас интересуют условия на вектор $d$ в терминах ограничения на отношения соседних его компонент, обеспечиваюшие вьполнение неравенства (3).

Введем матрицу перестановок

$$
P=\operatorname{circ}(0, \ldots, 0,1)
$$

Тогда любой циркулянт $A$ вида (2) может быть записан [6] следующим образом:

$$
A=P^{-n} Q_{A}(P),
$$

где

$$
Q_{A}(z)=z^{2 n}+a_{1} z^{2 n-1}+\cdots+a_{n} z^{n}+a_{n-1} z^{n-1}+\cdots+a_{1} z+1
$$

- многочлен, связанньй с матрищей $A$. Заметим, что многочлен $Q_{A}(z)$ удовлетворяет тождеству

$$
Q_{A}(z) \equiv z^{2 n} Q_{A}\left(\frac{1}{z}\right)
$$

Следовательно, справедливо разложение многочлена $Q_{A}(z)$ на множители

$$
\begin{gathered}
Q_{A}(z)=(z-1)^{m_{1}}(z+1)^{m_{2}}\left(z-z_{1}\right)\left(z-\frac{1}{z_{1}}\right) \cdots\left(z-z_{m}\right)\left(z-\frac{1}{z_{m}}\right) \\
m_{1}+m_{2}+2 m=2 n
\end{gathered}
$$

для некоторых, вообще говоря, комплексных чисел $z_{\nu}, \nu=1, \ldots, m$.

Сформулируем наш основной результат.

ТЕОРема 1. Пусть корни многочлена $Q_{A}(z)$, связанного с ииркулянтной матрицей $A$ вида (2), вещественны и среди них нет чисел \pm 1 . Тогда матрица $G A$ монотонна, где

$$
\begin{gathered}
G=G_{1} G_{2} \cdots G_{n}, \\
G_{\nu}= \begin{cases}-\operatorname{circ}\left(z_{\nu}+\frac{1}{z_{\nu}}, 1,0, \ldots, 0,1\right), & z_{\nu}<0 \\
-I, & z_{\nu}>0\end{cases}
\end{gathered}
$$

числа $z_{\nu}$ и $1 / z_{\nu}-$ корни многочлена $Q_{A}(z)$. 
ДоКАЗАТЕЛЬСТВо. Поскольку среди нулей многочлена $Q_{A}(z)$ нет чисел \pm 1 , то представление (4) будет иметь вид

$$
Q_{A}(z)=\left(z-z_{1}\right)\left(z-\frac{1}{z_{1}}\right)\left(z-z_{2}\right)\left(z-\frac{1}{z_{2}}\right) \cdots\left(z-z_{n}\right)\left(z-\frac{1}{z_{n}}\right) .
$$

Заметим, что

$$
\left(z-z_{\nu}\right)\left(z-\frac{1}{z_{\nu}}\right)=z^{2}-\left(z_{\nu}+\frac{1}{z_{\nu}}\right) z+1, \quad \nu=1, \ldots, n .
$$

Тогда

$$
Q_{A}(z)=\prod_{\nu=1}^{n}\left(z^{2}-\left(z_{\nu}+\frac{1}{z_{\nu}}\right) z+1\right) .
$$

Каждый многочлен второй степени (5) соответствует циркулянту

$$
A_{\nu}=\operatorname{circ}\left(-z_{\nu}-\frac{1}{z_{\nu}}, 1,0, \ldots, 0,1\right) \text {. }
$$

Таким образом, ленточная циркулянтная матрища $A$ вида (2) равна произведению трехдиагональных циркулянтов $A_{\nu}$, т.е. $A=A_{1} A_{2} \cdots A_{n}$.

Корни $z_{\nu}$ и $1 / z_{\nu}$ многочлена $Q_{A}(z)$ вещественны и отличны от \pm 1 , поэтому вьполнено неравенство

$$
\left|z_{\nu}+\frac{1}{z_{\nu}}\right|>2
$$

которое является условием диагонального преобладания матрицы (7). Следовательно, все $A_{\nu}, \nu=1, \ldots, n$, являются матрицами с диагональньм преобладанием.

Пусть корень $z_{\nu}$ многочлена $Q_{A}(z)$ отрицателен. В этом случае все элементы трехдиагональной матрищы $A_{\nu}$ неотрицательны. Для системы уравнений

$$
A_{\nu} x=d
$$

с такой матрицей применимо преобразование Мирошниченко [1], в результате которого получаем систему

$$
G_{\nu} A_{\nu} x=G_{\nu} d
$$

с монотонной матрицей $G_{\nu} A_{\nu}$, где

$$
G_{\nu}=-\operatorname{circ}\left(z_{\nu}+\frac{1}{z_{\nu}}, 1,0, \ldots, 0,1\right) .
$$

Если же корень $z_{\nu}$ многочлена $Q_{A}(z)$ положителен, то смена знаков элементов матрицы $A_{\nu}$ преврашает ее в монотонную. В этом случае $G_{\nu} A_{\nu}$ монотонна с

$$
G_{\nu}=-I
$$

т.e. $G_{\nu} A_{\nu}=-A_{\nu}$. 
Определим матрицу $G$ как произведение матриц

$$
G=G_{1} G_{2} \cdots G_{n}
$$

где матришы $G_{\nu}, \nu=1, \ldots, n$, задаются формулой (9) либо (10) в зависимости от знака корней $z_{\nu}$ многочлена $Q_{A}(z)$, соответствующего матрице $A$. Домножим исходное матричное уравнение (1) слева на матрищу $G$ :

$$
G A x=G d,
$$

и покажем, что матрица полученной системы (11) монотонна.

Систему уравнений (11) перепишем в виде

$$
G_{1} G_{2} \cdots G_{n} A_{1} A_{2} \cdots A_{n} x=G d
$$

Поскольку циркулянты являются перестановочными матрицами, то отсюда

$$
G_{1} A_{1} G_{2} A_{2} \cdots G_{n} A_{n} x=G d .
$$

Решение последней системы уравнений эквивалентно последовательному решению серии систем

$$
\left\{\begin{array}{l}
G_{1} A_{1} x_{1}=G d, \\
G_{2} A_{2} x_{2}=x_{1} \\
\ldots \ldots \ldots \ldots \ldots \ldots \ldots \ldots \ldots \ldots \\
G_{n-1} A_{n-1} x_{n-1}=x_{n-2}, \\
G_{n} A_{n} x=x_{n-1} .
\end{array}\right.
$$

По доказанному матришы $G_{\nu} A_{\nu}, \nu=1, \ldots, n$, являются монотонными; следовательно, условие $G d \geqslant 0$ обеспечивает вьполнение $x_{1} \geqslant 0$, а также $x_{\nu} \geqslant 0, \nu=2, \ldots, n-1$, и в конечном счете вьполнение условия $x \geqslant 0$. Таким образом, из неравенства $G d \geqslant 0$ следует неравенство $x \geqslant 0$, что и говорит о монотонности матрицы системы уравнений (11). Теорема 1 доказана.

Следуюшие две теоремы уточняют результат в случае корней многочлена $Q_{A}(z)$ одного знака.

ТЕОрема 2. Если корни многочлена $Q_{A}(z)$, связанного с ииркулянтной матриией $A$ вида (2), положсительны и $Q_{A}(1) \neq 0$, то матрица $(-1)^{n} A$ монотонна.

Теорема 3. Пусть корни многочлена $Q_{A}(z)$, связанного с ииркулянтной матрицей $A$ вида (2), отрицательны и $Q_{A}(-1) \neq 0$. Тогда матрица $G A$ монотонна, zde

$$
G=(-P)^{-n} Q_{A}(-P)
$$

m.e.

$$
G=(-1)^{n} \operatorname{circ}\left((-1)^{n} a_{n}, \ldots,-a_{1}, 1,0, \ldots, 0,1,-a_{1}, \ldots,(-1)^{n-1} a_{n-1}\right)
$$


ДокАЗАТЕЛЬСТВА этих теорем непосредственно вытекает из доказательства теоремы 1.

Теорема 2 (корни многочлена $Q_{A}(z)$, связанного с матрищей системы, только положительны) говорит о том, что решение системы уравнений (1) при неотрицательной правой части $d$ всегда будет либо неотрицательным ( $n$ четно), либо наоборот неположительным ( $n$ нечетно). Вопрос о положительности (неотрицательности) решения при положительной правой части $d$, если у многочлена $Q_{A}(z)$ есть отрицательные корни, не так тривиален. Мы даем ответ на этот вопрос в виде ограничений на отношение соседних компонент вектора правой части $d$.

Введем следующую характеристику матрицы $A$ :

$$
q^{*}(A)=\frac{Q_{A}(1)+(-1)^{n} Q_{A}(-1)}{Q_{A}(1)-(-1)^{n} Q_{A}(-1)} .
$$

Если все корни многочлена $Q_{A}(z)$ отрицательны, то на основании представления $(6)$ и неравенства (8) заключаем, что

$$
(-1)^{n} Q_{A}(-1)>0 \text {. }
$$

Таким образом, характеристику $q^{*}(A)$, определенную равенством $(12)$, можно записать так:

$$
q^{*}(A)=\frac{Q_{A}(1)+\left|Q_{A}(-1)\right|}{Q_{A}(1)-\left|Q_{A}(-1)\right|} .
$$

Поскольку в случае отрицательных корней многочлена $Q_{A}(z)$ его коэффициенты неотрицательны, то ясно, что

$$
q^{*}(A)>1
$$

ТЕОрема 4. Пусть все корни многочлена $Q_{A}(z)$, связанного с ииркулянтной матрицей $A$ вида (2), отрицательны и $Q_{A}(-1) \neq 0$. Вектор Gd будет положительным, если полохителен $d$, и его соседние компоненты отличаются не более чем в q раз при

$$
q<q^{*}(A)
$$

Доказательству предпошлем две леммы.

ЛЕмма 1. Пусть соседние компоненты положительного вектора $d$ отличаются не более чем в q раз. Тогда при

$$
\alpha_{\nu}>2 q
$$

компоненты вектора $G_{\nu} d$ также положительны, где

$$
G_{\nu}=\operatorname{circ}\left(\alpha_{\nu},-1,0, \ldots, 0,-1\right)
$$


ДокАЗАТЕЛЬСтво. Для отношения соседних компонент вектора $d$ введем обозначение $\lambda_{i}=d_{i} / d_{i+1}$. Тогда

$$
\left(G_{\nu} d\right)_{i}=-d_{i-1}+\alpha_{\nu} d_{i}-d_{i+1}=\left(-\lambda_{i-1}+\alpha_{\nu}+\frac{1}{\lambda_{i}}\right) d_{i} .
$$

Поскольку по условию величины $\lambda_{i-1}$ и $\lambda_{i}$ лежат в промежутке $[1 / q, q]$, то выражение $\left(G_{\nu} d\right)_{i}$ минимально при $\lambda_{i-1}=q$ и $\lambda_{i}=1 / q$ и, следовательно, всегда положительно при выполнении неравенства (14). Лемма 1 доказана.

ЛЕмма 2. Пусть выполнены условия леммы 1. Тогда отноиение соседних компонент вектора $G_{\nu} d$ не превосходит величинь

$$
\frac{\alpha_{\nu} q-2}{\alpha_{\nu}-2 q}
$$

ДокАЗАТЕЛЬСТво. Используя (15), запишем отношение соседних компонент вектоpa $G_{\nu} d$ :

$$
\frac{\left(G_{\nu} d\right)_{i}}{\left(G_{\nu} d\right)_{i+1}}=\frac{\left(-\lambda_{i-1}+\alpha_{\nu}+1 / \lambda_{i}\right) d_{i}}{\left(-\lambda_{i}+\alpha_{\nu}+1 / \lambda_{i+1}\right) d_{i+1}}=\frac{\lambda_{i}\left(-\lambda_{i-1}+\alpha_{\nu}\right)-1}{-\lambda_{i}+\alpha_{\nu}-1 / \lambda_{i+1}} .
$$

Ясно, что это выражение является убываюшей функцией по переменным $\lambda_{i+1}$ и $\lambda_{i-1}$ на интервале $[1 / q, q]$. Поэтому справедлива оценка

$$
\frac{\left(G_{\nu} d\right)_{i}}{\left(G_{\nu} d\right)_{i+1}} \leqslant \frac{\lambda_{i}\left(-1 / q+\alpha_{\nu}\right)-1}{-\lambda_{i}+\alpha_{\nu}-q} .
$$

А выражение справа максимально при $\lambda_{i}=q$. Итак,

$$
\frac{\left(G_{\nu} d\right)_{i}}{\left(G_{\nu} d\right)_{i+1}} \leqslant \frac{\alpha_{\nu} q-2}{\alpha_{\nu}-2 q}
$$

Лемма 2 доказана.

ДоКАЗАТЕЛЬСТВо ТЕОРЕМЫ 4. Доказательство проведем индукцией по $n$.

Пусть $n=1$. В этом случае матрица $A$ трехдиагональная:

$$
A=\operatorname{circ}\left(a_{1}, 1,0, \ldots, 0,1\right)
$$

соответственно $Q_{A}(z)=z^{2}+a_{1} z+1$. Справедливость теоремы следует из леммы 2 на основании эквивалентности неравенств (13) и (14). Здесь $G=G_{1}$ с $\alpha_{1}=a_{1}$ и

$$
\frac{Q_{A}(1)-Q_{A}(-1)}{Q_{A}(1)+Q_{A}(-1)}=\frac{a_{1}}{2} .
$$

Теперь предположим, что утверждение теоремы верно для $n=k$. Покажем, что тогда оно будет справедливым и для $n=k+1$.

Ясно, что многочлен $Q_{A}(z)$, связанный с матрицей $A$, можно представить в виде

$$
Q_{A}(z)=\widetilde{Q}(z)\left(z^{2}+\alpha_{k+1} z+1\right)
$$


где $\alpha_{k+1}=-z_{k+1}-1 / z_{k+1}>2$, а $z_{k+1}$ и $1 / z_{k+1}-$ некоторые из корней многочлена $Q_{A}(z)$. Многочлен $\widetilde{Q}(z)$ может быть связан с некоторой матрицей $\widetilde{A}$ вида $(2)$, его степень равна $2 k$. Матрищу, приводящую $\widetilde{A}$ к монотонной (см. теорему 3$)$, обозначим $\widetilde{G}$. Тогда $G=\widetilde{G} G_{k+1}$, где

$$
G_{k+1}=\operatorname{circ}\left(\alpha_{k+1},-1,0, \ldots, 0,-1\right) .
$$

Прежде всего заметим, что

$$
q<\frac{\alpha_{k+1}}{2}
$$

В самом деле, по условию теоремы $q<q^{*}(A)$, где согласно (12)

$$
q^{*}(A)=\frac{Q_{A}(1)+(-1)^{k+1} Q_{A}(-1)}{Q_{A}(1)-(-1)^{k+1} Q_{A}(-1)} .
$$

Учитьвая, что

$$
Q_{A}(1)=\left(\alpha_{k+1}+2\right) \widetilde{Q}(1), \quad Q_{A}(-1)=-\left(\alpha_{k+1}-2\right) \widetilde{Q}(-1),
$$

имеем

$$
q^{*}(A)=\frac{\widetilde{Q}(1)\left(\alpha_{k+1}+2\right)+(-1)^{k} \widetilde{Q}(-1)\left(\alpha_{k+1}-2\right)}{\widetilde{Q}(1)\left(\alpha_{k+1}+2\right)-(-1)^{k} \widetilde{Q}(-1)\left(\alpha_{k+1}-2\right)} .
$$

Поскольку $\alpha_{k+1}>2$ и $(-1)^{k} \widetilde{Q}(-1)<\widetilde{Q}(1)$, имеет место неравенство

$$
q^{*}(A)<\frac{\left(\alpha_{k+1}+2\right)+\left(\alpha_{k+1}-2\right)}{\left(\alpha_{k+1}+2\right)-\left(\alpha_{k+1}-2\right)}=\frac{\alpha_{k+1}}{2},
$$

которое и доказывает (16).

Неравенство (16) позволяет применить леммы 1 и 2 при $G_{\nu}=G_{k+1}$. В результате чего получаем, что компоненты вектора $\widetilde{d}=G_{k+1} d$ положительны и отношение соседних компонент этого вектора не превосходит величины

$$
\widetilde{q}=\frac{\alpha_{k+1} q-2}{\alpha_{k+1}-2 q}
$$

По предположению индукции вектор $\widetilde{G} \widetilde{d}$ будет положителен при выполнении условия

$$
\widetilde{q}<\frac{\widetilde{Q}(1)+(-1)^{k} \widetilde{Q}(-1)}{\widetilde{Q}(1)-(-1)^{k} \widetilde{Q}(-1)} .
$$

С учетом (18) последнее неравенство можно переписать в виде

$$
q<\frac{\left(\alpha_{k+1}+2\right) \widetilde{Q}(1)+(-1)^{k}\left(\alpha_{k+1}-2\right) \widetilde{Q}(-1)}{\left(\alpha_{k+1}+2\right) \widetilde{Q}(1)-(-1)^{k}\left(\alpha_{k+1}-2\right) \widetilde{Q}(-1)}
$$

которое, в свою очередь, согласно (17) совпадает с неравенством (13). Следовательно, вектор $\widetilde{G} \widetilde{d}=G d$ положителен. Теорема 4 полностью доказана. 
Отметим, что неравенство (13) является необходимым и достаточньг условием положительности вектора $G d$. Однако нельзя сказать, что оно является необходимым и для положительного решения системы (1), так как утверждение теоремы 3 носит лишь достаточньй характер.

В работе Киндалева [8] указано на связь величины $Q_{A}(-1)$ с нормой обратной матрицы к $A$ для рассматриваемого случая. Показано, что если матрица $A$ четного порядка, то

$$
\left\|A^{-1}\right\|=\frac{(-1)^{n}}{Q_{A}(-1)}
$$

для равномерной (и спектральной) нормы матрицы. Если же $A$ нечетного порядка, то величина $\| A^{-1}||$ достаточно близка к $\left|Q_{A}(-1)\right|^{-1}$ и справедлива оценка

$$
\left\|A^{-1}\right\|<\frac{(-1)^{n}}{Q_{A}(-1)} .
$$

Всегда можно считать, что матрица $A$ четного порядка (в силу цикличности $A$ число уравнений в системе (1) можно удвоить) и вьполнено равенство (19). В этом случае характеристику $q^{*}(A)$, определенную равенством $(12)$ и используемую в теореме 4 , можно выразить через число обусловленности матрицы $A$, определяемое как

$$
\operatorname{cond}(A)=\|A\| \cdot\left\|A^{-1}\right\|
$$

Имеем

$$
q^{*}(A)=\frac{\operatorname{cond}(A)+1}{\operatorname{cond}(A)-1} .
$$

Выражение (20) говорит о том, что для систем с плохо обусловленньми матрицами характеристика $q^{*}(A)$ близка к 1 и гарантировать положительность решения можно только при правых частях с “почти" одинаковыми компонентами.

В заключение рассмотрим применение полученных результатов к задачам периодической сплайн-интерполяпии на равномерной сетке. Пусть сплайн $S(x)$ степени $m$ минимального дефекта представлен в виде разложения по нормализованньп $B$-сплайнам

$$
S(x)=\sum_{j} \alpha_{j} B_{j}(x)
$$

При решении задачи интерполящии возникает система уравнений относительно коэффициентов $\alpha_{j}$ с ленточной матрищей, элементами которой являются $B_{j}\left(x_{i}\right)$, и правой частью, состоящей из интерполируемых значений $f_{i}, i=1, \ldots, N$, заданных в узлах $x_{i}$ равномерной сетки. Классическое определение интерполящионньх сплайнов четной и нечетной степени отличается (см. [9]) тем, что в первом случае узлы сплайна находятся между точками интерполящии, а во втором - совпадают с ними. Это приводит к тому, что ширина ленты матрицы будет $m+1$, если $m=2 n$ четно, и $m$, если $m=2 n+1$ нечетно, т.е. в обоих случаях ширина ленты системы

$$
\sum_{j=i-n}^{i+n} B_{j}\left(x_{i}\right) \alpha_{j}=f_{i}, \quad i=1, \ldots, N
$$


равна $2 n+1$. После умножения уравнений $(21)$ на нормирующий множитель

$$
\frac{1}{B_{i-n}\left(x_{i}\right)}= \begin{cases}m !, & m=2 n+1, \\ 2^{m} m !, & m=2 n\end{cases}
$$

матрица $A_{m}$ полученной системы будет иметь вид $(2)$, причем норма $A_{m}$ будет равна величине этого нормируюшего множителя, т.е.

$$
\left\|A_{m}\right\|= \begin{cases}m !, & m=2 n+1 \\ 2^{m} m !, & m=2 n\end{cases}
$$

поскольку сумма элементов любой строки матрицы системы (21) равна 1 и все эти элементы неотрицательны. Известно [9], что многочлены $Q_{A_{m}}(z)$, связанные с матрицами $A_{m}$, в обоих случаях имеют только отрицательные корни и, следовательно, вьполнены условия теорем 3 и 4 .

Таким образом, на основании теоремы 3 условия

$$
\sum_{j=i-n}^{i+n}(-1)^{i-j} B_{j}\left(x_{i}\right) f_{j} \geqslant 0, \quad i=1, \ldots, N
$$

гарантируют, что при интерполящии неотрицательных данных $f_{j}$ коэффищиенты $\alpha_{j}$ разложения сплайна по $B$-сплайнам будут неотрицательны, а значит, неотрицательным будет и сам сплайн $S(x)$. Вьпишем для примера условия (22) с числовьми коэффициентами для невысоких степеней - параболических, кубических, четвертой и пятой степеней, что соответствует $m=2,3,4$ и 5 .

Случай $m=2$. Параболический сплайн имеет вид

$$
-f_{i-1}+6 f_{i}-f_{i+1} \geqslant 0, \quad i=1, \ldots, N .
$$

Случай $m=3$. Кубический сплайн имеет вид

$$
-f_{i-1}+4 f_{i}-f_{i+1} \geqslant 0, \quad i=1, \ldots, N \text {. }
$$

Случай $m=4$. Сплайн четвертой степени имеет вид

$$
f_{i-2}-76 f_{i-1}+230 f_{i}-76 f_{i+1}+f_{i+2} \geqslant 0, \quad i=1, \ldots, N \text {. }
$$

Случай $m=5$. Сплайн пятой степени имеет вид

$$
f_{i-2}-26 f_{i-1}+66 f_{i}-26 f_{i+1}+f_{i+2} \geqslant 0, \quad i=1, \ldots, N .
$$

Чтобы воспользоваться теоремой 4, нам необходимо вычислить характеристику $q^{*}\left(A_{m}\right)$ матрищы $A_{m}$ либо по формуле (12), либо по формуле (20). Для сплайнов нечетной степени $m=2 n+1$ величина числа обусловленности матрицы $A_{m}$ найдена в работе [10]:

$$
\operatorname{cond}\left(A_{m}\right)=\frac{(m+1) !}{2^{m+1}\left(2^{m+1}-1\right)\left|B_{m+1}\right|}, \quad m=2 n+1
$$


где $B_{m+1}$ - числа Бернулли (см. [11]). Матрица для сплайнов четной степени $m=2 n$ рассматривались в статье [12], в которой вычислена норма обратной матрицы (сплайновая матрица в [12] отличается от нашей $A_{m}$ множителем $\left.2^{m}\right)$. Применительно к нашему случаю

$$
\left\|A_{m}^{-1}\right\|=\frac{1}{2^{m}\left|E_{m}\right|}, \quad m=2 n,
$$

где $E_{m}$ - числа Эйлера (см. [11]). Поэтому

$$
\operatorname{cond}\left(A_{m}\right)=\frac{m !}{\left|E_{m}\right|}, \quad m=2 n \text {. }
$$

Отметим, что в формулах (23) и (24) используются числа Бернулли и Эйлера только с четньми индексами (по определению такие числа с нечетньми индексами равны нулю).

ТАБЛИЦА

\begin{tabular}{|c|c|c|}
\hline$m$ & cond $\left(A_{m}\right)$ & $q^{*}\left(A_{m}\right)$ \\
\hline \hline 2 & 2 & 3 \\
3 & 3 & 2 \\
4 & 4.8 & 1.53 \\
5 & 7.5 & 1.31 \\
6 & 11.80 & 1.19 \\
7 & 18.53 & 1.11 \\
8 & 29.11 & 1.07 \\
9 & 45.73 & 1.04 \\
10 & 71.83 & 1.03 \\
11 & 112.83 & 1.02 \\
\hline
\end{tabular}

В таблице приведены числовые значения $\operatorname{cond}\left(A_{m}\right)$ обусловленности матрицы $A_{m}$ и ее характеристики $q^{*}\left(A_{m}\right)$ для сплайнов до 11 -й степени, т.е. $m=2,3, \ldots, 11$. Значения $\operatorname{cond}\left(A_{m}\right)$ для нечетных $m$, приводимых в таблице, имеются в работе [10]. Там же отмечено, что для больших (нечетных) $m$, привлекая известные свойства чисел Бернулли, можно показать

$$
\operatorname{cond}\left(A_{m}\right) \approx \frac{1}{2}\left(\frac{\pi}{2}\right)^{m+1}
$$

Оказывается, асимптотика (25) остается справедливой и для больших четных $m$. Действительно, для чисел $E_{2 k}$ известны двухсторонние оценки [11]

$$
\frac{4^{k+1}(2 k) !}{\pi^{2 k+1}\left(1+3^{-1-2 k}\right)}<\left|E_{2 k}\right|<\frac{4^{k+1}(2 k) !}{\pi^{2 k+1}}, \quad k=0,1, \ldots
$$

применение которых к (24) и дает (25).

Вычисления показывают, что, уже начиная со сплайнов шестой степени $(m=6)$, значения cond $\left(A_{m}\right)$, получаемые по формуле $(25)$, совпадают со значениями $\operatorname{cond}\left(A_{m}\right)$ таблицы с точностью до всех приводимых знаков. 
Выражение (25) говорит о том, что с ростом степени сплайнов $m$ экспоненциально растет обусловленность сплайновых матриц. При этом характеристики $q^{*}\left(A_{m}\right)$ матриц $A_{m}$ быстро стремятся к 1 (что и отражено в таблице). Чтобы на основании теоремы 4 гарантировать положительность сплайна высокой степени при интерполяции положительных функций, необходимо загустить сетку так, чтобы отношение соседних интерполируемых значений не превосходило характеристику $q^{*}\left(A_{m}\right)$. Ясно, что для непрерьвных функций этого всегда можно добиться. Однако, если интерполируемая функция имеет участки с большими градиентами, то шаг сетки необходимо выбирать достаточно мальм, что ведет к увеличению объема информации и потере точности при численном решении задач интерполящии.

ЗАмечАниЕ. Доказанные теоремы переносятся и на случай бесконечных матриц, что соответствует кардинальной сплайн-интерполящии.

\section{СПИСОК ЦИТИРОВАННОЙ ЛИТЕРАТУРЫ}

[1] Miroshnichenko V. L. Convex and monotone spline interpolation // Constructive Theory of Functions'84. Proceed. Intern. Conf. Varna, Sofia: Publ. House of Bulgarian Acad. of Sci., 1984. P. 610-620.

[2] Верлан И. И. Консервативная интерполяция обобщенными сплайнами. Автореферат дисс. ‥ к. ф.-м. н. М.: МГУ, 1989.

[3] Завьялов Ю.С. Монотонная интерполяция обобщенньми кубическими сплайнами класса $C^{2}$ // Интерполяция и аппроксимация сплайнами. Вып. 147. Вычислительные системы. Новосибирск: ИМ СО РАН, 1992. С. 44-67.

[4] Завьялов Ю. С. Выпуклая интерполяция обобщенньми кубическими сплайнами класса $C^{2}$ // Сплайны и их приложения. Вып. 154. Вычислительные системы. Новосибирск: ИМ СО $\mathrm{PAH}, 1995$. C. $15-64$.

[5] Завьялов Ю.С. О неотрицательном решении системы уравнений с нестрого якобиевой матрицей // Сиб. матем. ж. 1996. Т. 37. №6. С. 1303-1307.

[6] Маркус М., Минк Х. Обзор по теории матриц и матричных неравенств. М.: Наука, 1972.

[7] Алберг Дж., Нильсон Э., Уолш Дж. Теория сплайнов и ее приложения. М.: Мир, 1972.

[8] Киндалев Б. С. Точная оценка нормы обратной матрицы для симметрического циркулянта // Аппроксимация сплайнами. Вып. 121. Вычислительные системы. Новосибирск: ИМ СО AH CCCP, 1987. C. $37-45$.

[9] Стечкин С. Б., Субботин Ю.Н. Сплайны в вычислительной математике. М.: Наука, 1976.

[10] Albasiny E. L., Hoskins W. D. Explicit error bounds for periodic splines of odd order on a uniform mesh // J. Inst. Math. Appl. 1973. V. 12. № 3. P. 303-318.

[11] Абрамовиц М., Стиган И. (ред. ) Справочник по специальным функциям. М.: Наука, 1979.

[12] Киндалев Б. С. Асимптотика погрешности и суперсходимость периодических интерполяционных сплайнов четной степени // Сплайны в вычислительной математике. Вып. 115. Вычислительные системы. Новосибирск: ИМ СО АН СССР, 1986. С. 3-25. 